\title{
Synthesis of New Anthracycline Derivatives Containing Lactic or Stearic Acid Moiety
}

\author{
Young S. Rho, Wan-Joong Kim, and Dong Jin Yoo," \\ Deparment of Chemistry, Chonbuk National University, Jeoniu 561-756, Korea \\ 'Department of Chemistry, Seonam University, Namwon 590-711, Korea. "E-mail: diyoo@seonam.ac.kr \\ Received July 1, 2006
}

\begin{abstract}
Novel anthracycline analogues 2-9 as potential anticancer agents were synthesized from daunomycin (1a) and doxorubicin (1b). Compounds 2, 6, and 7 were prepared by the nucleophilic displacement type esterification of a 14-bromodaunomycin (1c) with a sodium lactate, and stearic acid, respectively. Compounds 3-5 and 7-9 were prepared by the reaction of either daunomycin (1a) or doxorubicin (1b) with L-lactic and stearic acids in the presence of EDCI/PP reagents.
\end{abstract}

Key Words : Daunomycin, Doxorubicin, Anthracycline derivatives, Lactic acid, Stearic acid

\section{Introduction}

The anthracycline antibiotics, daunomycin (1a) and doxorubicin (1b), are the member of a very important class of cytotoxic agent that has been used for many years in the treatment of many different types of cancer. Doxorubicin (1b), a well known anticancer drug, is considered as one of the most active single anticancer agents because of its broad antitumour spectrum. ${ }^{1}$ Chemotherapy with doxorubicin, however, is largely limited by the cumulative dose-related cardiotoxicity, resulting in congestive heart failure. It is generally believed that the formation of free radicals plays a crucial role, and the involvement of radicals in the mechanism of doxorubicin-induced cardiotoxicity has been the subject of extensive reviewing ${ }^{3}$ (Figure 1).

As a result, numerous synthetic efforts have been devoted to overcome these disadvantages, culminating in the development of daunomycin (1a) or doxorubicin (1b) derivatives. ${ }^{4-7}$ Many examples of coupling daunomycin (1a) or doxorubicin (1b) with some amino acids ${ }^{\gamma-13}$ as well as blending $1 \mathrm{a}$ or $1 \mathrm{~b}$ with some amino acids ${ }^{4}$ are reported.

L-Lactic acid occurs in small quantities in the blood and muscle fluid of man and animals. The lactic acid concentration increases in muscle and blood after vigorous activity.

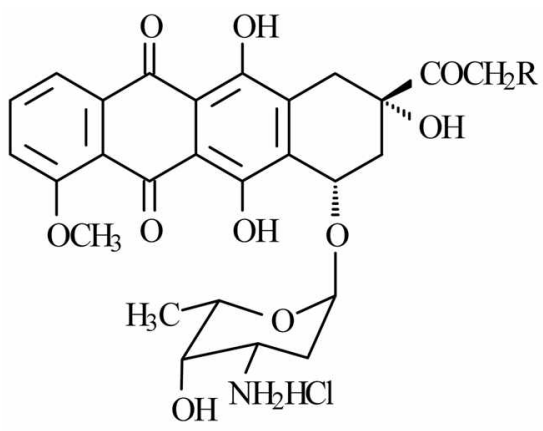

1a. Daunomycin $\mathrm{R}=\mathrm{H}$

1b. Doxorubicin $\mathrm{R}=\mathrm{OH}$

Figure 1. Chemical structures of daunomycin and doxorubicin.
In muscle, pyruvic acid is reduced to lactic acid during exertion. Stearic acid is also a promising material having strong adsorption property in a cell wall. ${ }^{1+}$ Therefore, anthracyclines containing a lactic or stearic acid residue are expected to be good potential delivery drugs, maybe due to diminishing cardiotoxicity and undesirable side effects. Herein, we describe the synthesis of new anthracycline derivatives via coupling of $\mathrm{C}_{14}-\mathrm{OH}$ and $\mathrm{C}_{3}-\mathrm{NH}$, in DM (1a) and DX (1b) with two kinds of acid molecules, L-lactic and stearic acid.

\section{Results and Discussion}

In the previous papers, we described the total synthesis of anthracyclinone derivatives through Michael type condensation $^{15-18}$ or Friedel-Crafts acylation. ${ }^{1920}$ We reported the successful preparation of a new aglycone containing an ester linkage at C-14 through a nucleophilic displacement esterification method. ${ }^{1921}$ In more recent papers, we have also reported the successful synthesis of some new anthracycline analogues from commercially available anticancer agents, such as daunomycin (1a) and doxorubicin (1b). ${ }^{8-13}$ Several new anthracycline derivatives were synthesized using acylation methods (Scheme 1). The synthesis of 14-bromo DM (1c) from 1a was accomplished by the known procedure. ${ }^{921-23}$ All compounds 2-9 were obtained through acylation of the $\mathrm{C}-14$ hydroxyl group in the aglycon and/or the amino group at $\mathrm{C}-3^{\prime}$ in the glycone with L-lactic acid and stearic acid.

DM-lac (2) and DM-st (6), potential prodrugs, were prepared by the reaction of 14-bromo DM (1c) with a sodjum lactate or stearic acid. For the purpose of comparing the activity of $\mathbf{2}$ and $\mathbf{6}$, carboamidation compounds, DMNlac (3), DX-Nlac (4), DM-Nst (7), and DX-Nst (8) were synthesized by amidation of the amino group at $\mathrm{C}-3^{\prime}$ of sugar moiety in 1a or 1b with the corresponding acids. In addition, $N$-acylation compounds, DXlac-Nlac (5) and DXst-Nst (9), were prepared through esterification of the $\mathrm{C}_{14}-\mathrm{OH}$ in $\mathrm{DX}$ (1b) with the corresponding acids followed by amidation of the amino group at the sugar moiety with the corresponding 


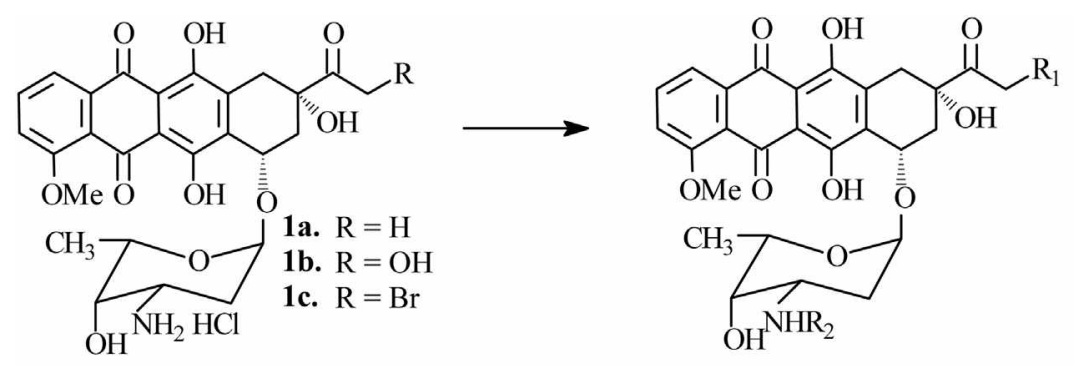
2. $\mathrm{R}_{1}=\mathrm{V}, \mathrm{R}_{2}=\mathrm{HHCl}$ (DM-lac)
3. $\mathrm{R}_{1}=\mathrm{H}, \mathrm{R}_{2}=\mathrm{W}$ (DM-Nlac)
4. $\mathrm{R}_{\mathrm{I}}=\mathrm{OH}, \mathrm{R}_{2}=\mathrm{W}(\mathrm{DX}-\mathrm{Nlac})$
5. $\mathrm{R}_{1}=\mathrm{V}, \mathrm{R}_{2}=\mathrm{W}$ (DXlac-Nlac)
6. $\mathbf{R}_{1}=\mathrm{X}, \mathrm{R}_{2}=\mathrm{HHCl}$ (DM-st)
7. $R_{1}=H, R_{2}=Y \quad$ (DM-Nst)
8. $R_{1}=O H, R_{2}=Y$ (DX-Nst)
9. $R_{1}=X, R_{2}=Y \quad$ (DXst-Nst)

Scheme 1. Synthesis of new anthracycline analogues 2-9.

acids.

First, 14-bromo DM (1c) was synthesized in best yield when a minimum quantity of co-solvent (methanol/1,4dioxane, $\mathrm{v} / \mathrm{v}=1: 2$ ) was used, because it diminished the formation of side product from dimethylketalization of ketone at $\mathrm{C}-13$. The rate of bromination depended on the reaction temperature and time. The optimal conditions are bromination at $30^{\circ} \mathrm{C}$ for $40 \mathrm{~min}$.

DM-lac (2) was synthesized as follows: To a 14-bromo DM (1c) prepared by introducing $\mathrm{Br}$ atom at C-14 of daunomycine (1a) was added a solution of sodium lactate in acetone; the mixture was heated at reflux at for $4 \mathrm{hr}$. After removing the solvent under reduced pressure, the residue was dissolved in $\mathrm{THF}$ and treated with etheral $\mathrm{HCl}$ followed by stirring at $-20^{\circ} \mathrm{C}$ for $2 \mathrm{hr}$ and further stirring at room temperature for $3 \mathrm{hr}$ to give DM-lac (2). DM-st (6) was synthesized from DM (1c) and stearic acid in triethylamine/ acetone as described for the preparation of DM-lac (2).

Many attempts to prepare DM-Nlac (3) and DX-Nlac (4) through direct coupling of the amino group in daunomycin (1a) or doxorubicin (1b) with L-lactic acid using 1,3dicyclohexylcarbodimide (DCC)/4-(dimethylamino) pyridine (DMAP) $)^{24}$ failed. Reactants $(\mathbf{1} \mathbf{a}, \mathbf{1 b})$ and DCU (djcyclohexylurea) were observed as main products. Eventually, DM-Nlac (3) was synthesized by coupling of the $\mathrm{NH}_{2} \mathrm{HCl}$ in 1a with L-lactic acid using 1-ethyl-3-(3dimethylaminopropyl)carbodiimide (EDCI) in the presence of catalytic amounts of 4-pyrrolidinopyridine (PP). ${ }^{25,26} \mathrm{DX}$ Nlac (4) was synthesized from DX (1b) as described for the preparation of DM-Nlac (3). For the reaction of $1 \mathrm{~b}$, however, the competition between the C-14 hydroxyl group and the amine group at $\mathrm{C}-3^{\prime}$ was observed. Both products of DXNlac (4) and DXlac-Nlac (5) were formed and the product ratio depended on the amounts of L-lactic acid and EDCI used in the reaction. DXlac-Nlac (5) was prepared using 2.2 equivalent of the corresponding acid and EDCI under the

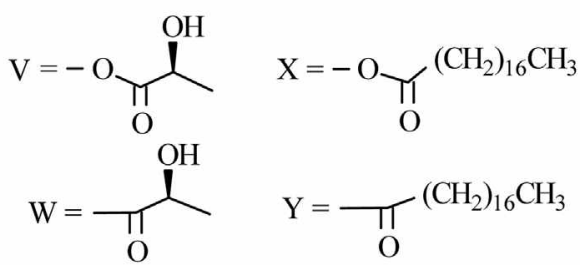

same conditions.

Synthesis of DM-Nst (7) and DX-Nst (8) was carried out as follows: stearic acid and EDCI (1.2 equiv) was dissolved in dry DMF and the mixture was stirred at $0^{\circ} \mathrm{C}$ for $30 \mathrm{~min}$; to the reaction mixture was added DM (1a) or DX (1b) and catalytic amounts of PP, and the mixture was then stirred at room temperature for $3 \mathrm{hr}$ to give DM-Nst (7) and DX-Nst (8). DXst-Nst (9) was synthesized from $1 \mathrm{~b}$ as described for the preparation of 8 by increasing the amounts of stearic acid (2.2 equiv) and $\mathrm{EDCI}$ ( 2.2 equiv).

In conclusion, we synthesized the new anthracycline analogues including lactic or stearic acid as potential anticancer agents. Detailed biological tests of the title glycosides in vitro will be reported elsewhere in the future.

\section{Experimental Section}

All reactions were carried out under argon atmosphere in dried glassware. All solvents were carefully dried and distilled as reported. ${ }^{27}$ Bulk grade hexane was distilled before use. Merck pre-coated silica gel plates (Art.5554) with fluorescent indicator were used as analytical TLC. Gravity column chromatography and flash column chromatography were carried out on silica gel $(230-400$ mesh from Merck). 'H and ${ }^{13} \mathrm{C}$ NMR spectra were recorded on a JEOL JNM EX-400 spectrometer. Chemical shifts were intemally referenced to TMS for ${ }^{1} \mathrm{H}$ or to solvent signals for ${ }^{13} \mathrm{C}$. Infrared spectra were recorded on a Nicolet 5-DXB series FT-IR spectrophotometer. Mass spectra were obtained on a JEOL JMS HX-110/110A Tandem mass spectrometer $\left(\mathrm{FAB}^{+}, \mathrm{ESI}\right)$. UV-VIS absorption spectra were recorded on a Hitachi-556 spectrophotometer. Optical rotations were determined using the Rudolph AUTOPOL IV apparatus with a $0-100-1.5$ polarimeter sample tube. Melting points were obtained on a Büchi 510 melting point apparatus and are uncorrected. 
Daunomycin-14-lactate hydrochloride (2). A solution of 14-bromodaunomycin hydrochloride $(1 \mathrm{c}, 0.30 \mathrm{~g}, 0.46$ $\mathrm{mmol})$ and sodium lactate $(0.10 \mathrm{~g}, 0.93 \mathrm{mmol})$ in acetone $(300 \mathrm{~mL})$ was heated at reflux for $4 \mathrm{hr}$. Upon completion of the reaction the solvent was evaporated. The residue was dissolved in dry THF $(150 \mathrm{~mL})$, etheral $\mathrm{HCl}$ was added, and the mixture was stirred at $-20^{\circ} \mathrm{C}$ for $2 \mathrm{hr}$ and further stirred at room temperature for $3 \mathrm{hr}$. The organic solvent was concentrated by a rotary evaporator and the residue was purified by column chromatography on silica gel $\left(\mathrm{CH}_{2} \mathrm{Cl}_{2} /\right.$ $\mathrm{CH}_{3} \mathrm{OH} / \mathrm{HCO}_{2} \mathrm{H} / \mathrm{H}_{2} \mathrm{O}=88: 15: 2: 1$ ) to give daunomycin14-lactate hydrochloride $(2,0.29 \mathrm{~g}, 97 \%)$ as a red powder: mp 120-122 ${ }^{\circ} \mathrm{C} ;[\alpha]_{\mathrm{D}}^{20}+110.98^{\circ}\left(\mathrm{c} 0.1, \mathrm{CH}_{3} \mathrm{OH}\right) ; \mathrm{IR}(\mathrm{KBr})$ $3432,2939,1793,1738,1609,1387,1283,1215,1129,987$, $793 \mathrm{~cm}^{-1} ;{ }_{1}^{1} \mathrm{H}$ NMR $\left(400 \mathrm{MHz}, \mathrm{DMSO}-d_{6}\right) \delta 13.91(\mathrm{~s}, 1 \mathrm{H}$, $\mathrm{PhOH}), 13.24$ (s, 1H, $\mathrm{PhOH}), 8.29\left(\mathrm{~s}, 2 \mathrm{H}, \mathrm{NH}_{2}\right), 7.78(\mathrm{~m}$, $2 \mathrm{H}, \mathrm{ArH}), 7.55(\mathrm{~m}, 1 \mathrm{H}, \mathrm{ArH}), 5.60\left(\mathrm{~d}, 1 \mathrm{H}, J=6.3 \mathrm{~Hz}, \mathrm{C}_{4} \mathrm{H}\right.$ ), $5.50\left(\mathrm{~s}, 1 \mathrm{H}, \mathrm{C}_{9} \mathrm{OH}\right), 5.28\left(\mathrm{~d}, 1 \mathrm{H}, J=18.0 \mathrm{~Hz}, \mathrm{C}_{14} \mathrm{H}\right), 5.11(\mathrm{~d}$, $\left.1 \mathrm{H}, J=3.9 \mathrm{~Hz}, \mathrm{C}_{7 \mathrm{eq}} \mathrm{H}\right), 5.28\left(\mathrm{~d}, 1 \mathrm{H}, J=18.0 \mathrm{~Hz}, \mathrm{C}_{14} \mathrm{H}\right), 4.84$ $\left(\mathrm{m}, 1 \mathrm{H}, \mathrm{C}_{1} \mathrm{H}\right), 4.16\left(\mathrm{q}, 1 \mathrm{H}, J=6.3 \mathrm{~Hz}, \mathrm{C}_{5} \mathrm{H}\right), 4.03(\mathrm{q}, 1 \mathrm{H}, J$ $\left.=6.8 \mathrm{~Hz}, \mathrm{C}_{16} \mathrm{H}\right), 3.93\left(\mathrm{~s}, 3 \mathrm{H}, \mathrm{C}_{4} \mathrm{OCH}_{3}\right), 3.63(\mathrm{~m}, 1 \mathrm{H}$, $\left.\mathrm{C}_{4} \mathrm{OH}\right), 3.32\left(\mathrm{~m}, 1 \mathrm{H}, \mathrm{C}_{3} \mathrm{H}\right), 3.04(\mathrm{~d}, 1 \mathrm{H}, J=17.5 \mathrm{~Hz}$, $\left.\mathrm{C}_{10 \mathrm{eq}} \mathrm{H}\right), 2.73\left(\mathrm{~d}, 1 \mathrm{H}, J=17.5 \mathrm{~Hz}, \mathrm{C}_{10 \mathrm{ax}} \mathrm{H}\right), 2.20(\mathrm{~d}, 1 \mathrm{H}, J=$ $\left.14.6 \mathrm{~Hz}, \mathrm{C}_{8 \mathrm{eq}} \mathrm{H}\right), 2.09\left(\mathrm{dd}, 1 \mathrm{H}, J=3.9,14.6 \mathrm{~Hz}, \mathrm{C}_{82 \mathrm{X}} \mathrm{H}\right.$ ), $1.67-1.90\left(\mathrm{~m}, 2 \mathrm{H}, \mathrm{C}_{2} \mathrm{H}\right), 1.19\left(\mathrm{~d}, 3 \mathrm{H}, J=6.1 \mathrm{~Hz}, \mathrm{C}_{5} \mathrm{CH}_{3}\right)$, 1.11 (d, $\left.3 \mathrm{H}, J=6.8 \mathrm{~Hz}, \mathrm{C}_{1}, \mathrm{CH}_{3}\right) ;{ }^{13} \mathrm{C}$ NMR $(100 \mathrm{MHz}$, DMSO-d $\left.d_{6}\right) \delta 208.13,186.14,183.31,180.57,174.18$, $165.05,160.71,136.16,135.07,134.54,134.07,119.74$, $118.89,110.58,99.30,75.02,69.43,66.70,66.15,65.98$, $65.77,56.57,50.67,46.52,35.94,31.82,28.99,28.29$, 20.59. 16.70; UV $\left(\mathrm{CH}_{3} \mathrm{OH}\right): \lambda_{\max }(\log \varepsilon)=205(0.19), 249$ $(0.05), 485(0.03)$; Mass $\left(\mathrm{FAB}^{+}, \mathrm{Na}\right) \mathrm{m} / \mathrm{z} 639(\mathrm{M}-\mathrm{HCl}+$ $\mathrm{Na})^{+}$.

Daunomycin-3'- $N$-lacticcarboamide (3). The mixture of L-lactic acid $(0.08 \mathrm{~mL}, 1.06 \mathrm{mmol})$ and EDCI $(0.20 \mathrm{~g}, 1.06$ $\mathrm{mmol})$ in dry DMF $(80 \mathrm{~mL})$ was stirred on an ice bath for 30 $\mathrm{min}$ and allowed to warm to room temperature. To the stirred solution was added daunomycin hydrochloride $(1 \mathrm{a}, 0.50 \mathrm{~g}$, $0.88 \mathrm{mmol}$ ) and catalytic amounts of 4-pyrrolidinopyridine, and the mixture was stirred then for $12 \mathrm{hr}$. The reaction mixture was dissolved in $\mathrm{CH}_{2} \mathrm{Cl}_{2}(200 \mathrm{~mL})$, washed with water $(2 \times 200 \mathrm{~mL})$ and brine $(2 \times 200 \mathrm{~mL})$, dried over $\mathrm{MgSO}_{4}$, and the solvent was removed under reduced pressure. The residue was purified by column chromatography on silica gel $\left(\mathrm{CH}_{2} \mathrm{Cl}_{2} / \mathrm{Hexane} / \mathrm{CH}_{3} \mathrm{OH}=8: 1: 1\right)$ to give daunomycin- $3^{-}-N$-lacticcarboamide $(3,0.50 \mathrm{~g}, 94 \%)$ as a red powder: $\mathrm{mp} 127-129^{\circ} \mathrm{C} ;[\alpha]_{\mathrm{D}}^{20}+120.01^{\circ}\left(\mathrm{c} 0.1, \mathrm{CH}_{3} \mathrm{OH}\right.$ ); IR (KBr) 3399, 2927, 2855, 1716, 1622, 1580, 1529, 1411, $1289,1236,1207,1119,985,821,760,620 \mathrm{~cm}^{-1}$; 'H NMR $\left(400 \mathrm{MHz}, \mathrm{CDCl}_{3}\right) \delta 13.95(\mathrm{~s}, 1 \mathrm{H}, \mathrm{PhOH}), 13.21(\mathrm{~s}, 1 \mathrm{H}$, $\mathrm{PhOH}$ ), 7.99 (d, $1 \mathrm{H}, J=7.8 \mathrm{~Hz}, \mathrm{ArH}), 7.76$ (dd, $1 \mathrm{H}, J=7.8$, $8.3 \mathrm{~Hz}, \mathrm{ArH}), 7.35$ (d, $1 \mathrm{H}, J=8.3 \mathrm{~Hz}, \mathrm{ArH}), 6.90(\mathrm{~d}, 1 \mathrm{H}, J=$ $8.3 \mathrm{~Hz}, \mathrm{NH}), 5.49\left(\mathrm{~s}, 1 \mathrm{H}, \mathrm{C}_{9} \mathrm{OH}\right), 5.16(\mathrm{~d}, 1 \mathrm{H}, J=5.8 \mathrm{~Hz}$, $\left.\mathrm{C}_{4} \mathrm{H}\right), 4.90\left(\mathrm{~d}, 1 \mathrm{H}, J=3.9 \mathrm{~Hz}, \mathrm{C}_{7 \mathrm{cq}} \mathrm{H}\right), 4.48\left(\mathrm{~m}, 1 \mathrm{H}, \mathrm{C}_{1} \mathrm{H}\right)$, 4.09-4.22 (m, 2H, $\left.\mathrm{C}_{5} \mathrm{H}, \mathrm{Nlac} \alpha \mathrm{H}\right), 4.03\left(\mathrm{~s}, 3 \mathrm{H}, \mathrm{C}_{4} \mathrm{OCH}_{3}\right)$, $3.66\left(\mathrm{~m}, 1 \mathrm{H}, \mathrm{C}_{4} \mathrm{OH}\right), 3.23\left(\mathrm{~m}, 1 \mathrm{H}, \mathrm{C}_{3} \mathrm{H}\right), 3.19(\mathrm{~d}, 1 \mathrm{H}, J=$ $\left.19.0 \mathrm{~Hz}, \mathrm{C}_{10 \mathrm{eg}} \mathrm{H}\right), 2.86\left(\mathrm{~d}, 1 \mathrm{H}, J=19.0 \mathrm{~Hz}, \mathrm{C}_{10 a x} \mathrm{H}\right), 2.41$ (s, $\left.3 \mathrm{H}, \mathrm{C}_{14} \mathrm{CH}_{3}\right), 2.29\left(\mathrm{~d}, 1 \mathrm{H}, J=14.1 \mathrm{~Hz}, \mathrm{C}_{8 \mathrm{eq}} \mathrm{H}\right), 2.04(\mathrm{dd}, 1 \mathrm{H}$, $\left.J=3.9,14.1 \mathrm{~Hz}, \mathrm{C}_{8 \alpha x} \mathrm{H}\right), 1.85\left(\mathrm{~m}, 2 \mathrm{H}, \mathrm{C}_{2} \mathrm{H}\right), 1.32(\mathrm{~d}, J=6.8$ $\left.\mathrm{Hz}, 3 \mathrm{H}, \mathrm{C}_{5} \mathrm{CH}_{3}\right), 1.27\left(\mathrm{~d}, 1 \mathrm{H}, J=7.3 \mathrm{~Hz}\right.$, Nlac $\left.\beta \mathrm{CH}_{3}\right) ;{ }^{13} \mathrm{C}$ $\mathrm{NMR}\left(100 \mathrm{MHz}, \mathrm{CDCl}_{3}\right) \delta 212.05,186.95,186.53,174.29$, $160.93,156.41,155.74,135.67,135.42,134.37,134.03$, $120.76,119.75,118.34,111.38,111.16,100.59,76.57$, $69.92,69.25,68.40,66.97,56.58,45.15,35.03,33.28$, $29.67,24.85,21.01,16.74 ; \mathrm{UV}\left(\mathrm{CH}_{3} \mathrm{OH}\right): \lambda_{\max }(\log \varepsilon)=235$ (0.32), $252(0.20), 482(0.99)$; Mass $\left(\mathrm{FAB}^{+}, \mathrm{Na}\right) \mathrm{m} / \mathrm{z} 623$ (M $+\mathrm{Na})^{+}$.

Doxorubicin-3'- $\mathrm{N}$-lacticcarboamide (4). The mixture of L-lactic acid $(0.09 \mathrm{~mL}, 1.24 \mathrm{mmol})$ and EDCI $(0.24 \mathrm{~g}, 1.24$ mmol) in dry DMF $(100 \mathrm{~mL})$ was stirred on an ice bath for $30 \mathrm{~min}$ and allowed to warm to room temperature. To the stirred solution was added doxorubicin hydrochloride (1), $0.60 \mathrm{~g}, 1.03 \mathrm{mmol}$ ) and catalytic amounts of 4-pyrrolidinopyridine, and the mixture was then stirred for $14 \mathrm{hr}$. The reaction mixture was dissolved in $\mathrm{CH}_{2} \mathrm{Cl}_{2}(200 \mathrm{~mL})$, washed with water $(2 \times 200 \mathrm{~mL})$ and brine $(2 \times 200 \mathrm{~mL})$, dried over $\mathrm{MgSO}_{4}$, and the solvent was removed under reduced pressure. The residue was purified by column chromatography on silica gel $\left(\mathrm{CH}_{2} \mathrm{Cl}_{2} / \mathrm{Hexane} / \mathrm{CH}_{3} \mathrm{OH}=8: 1: 1\right)$ to give doxorubicin-3'- $N$-lacticcarboamide $(4,0.59 \mathrm{~g}, 92 \%)$ as a red powder: mp $116-117^{\circ} \mathrm{C} ;[\alpha]_{\mathrm{D}}^{20}+72.01^{\circ}\left(\mathrm{c} 0.1, \mathrm{CH}_{3} \mathrm{OH}\right)$; IR (KBr) $3425,2926,2254,2128,1728,1658,1580,1530$, $1441,1411,1377,1286,1241,1118,1020,992,822,764$, $708,624 \mathrm{~cm}^{-1} ;{ }^{1} \mathrm{H} \mathrm{NMR}\left(400 \mathrm{MHz}, \mathrm{CDCl}_{3}\right) \delta 13.95(\mathrm{~s}, 1 \mathrm{H}$, $\mathrm{PhOH}$ ), 13.24 (s, 1H, PhOH), 8.00 (d, 1H, $J=7.8 \mathrm{~Hz}, \mathrm{ArH}$ ) 7.78 (dd, $1 \mathrm{H}, J=7.8,8.3 \mathrm{~Hz}, \mathrm{ArH}), 7.40(\mathrm{~d}, 1 \mathrm{H}, J=8.3 \mathrm{~Hz}$, $\operatorname{ArH}), 7.06(\mathrm{~d}, 1 \mathrm{H}, J=8.7 \mathrm{~Hz}, \mathrm{NH}), 5.51(\mathrm{~d}, 1 \mathrm{H}, J=5.8 \mathrm{~Hz}$, $\left.\mathrm{C}_{4} \mathrm{H}\right), 5.24\left(\mathrm{~s}, 1 \mathrm{H}, \mathrm{C}_{9} \mathrm{OH}\right), 4.95\left(\mathrm{~d}, 1 \mathrm{H}, J=3.9 \mathrm{~Hz}, \mathrm{C}_{7 \mathrm{eq}} \mathrm{H}\right)$, $4.83\left(\mathrm{~m}, 1 \mathrm{H}, \mathrm{C}_{1} \mathrm{H}\right), 4.77\left(\mathrm{~s}, 2 \mathrm{H}, \mathrm{C}_{14} \mathrm{H}\right), 4.09-4.30(\mathrm{~m}, 2 \mathrm{H}$, $\left.\mathrm{C}_{5} \mathrm{H}, \mathrm{Nlac} \alpha \mathrm{H}\right), 4.07\left(\mathrm{~s}, 3 \mathrm{H}, \mathrm{C}_{4} \mathrm{OCH}_{3}\right), 3.62\left(\mathrm{~m}, 1 \mathrm{H}, \mathrm{C}_{4} \mathrm{OH}\right)$, $3.31\left(\mathrm{~m}, 1 \mathrm{H}, \mathrm{C}_{3} \mathrm{H}\right), 3.23\left(\mathrm{~d}, 1 \mathrm{H}, J=18.5 \mathrm{~Hz}, \mathrm{C}_{10 \mathrm{eg}} \mathrm{H}\right), 2.98$ (d, $1 \mathrm{H}, J=18.5 \mathrm{~Hz}, \mathrm{C}_{10 \mathrm{ax}} \mathrm{H}$ ), 2.37 (d, $1 \mathrm{H}, J=14.1 \mathrm{~Hz}$, $\left.\mathrm{C}_{8 \mathrm{eq}} \mathrm{H}\right), 2.15\left(\mathrm{dd}, 1 \mathrm{H}, J=3.9,14.1 \mathrm{~Hz}, \mathrm{C}_{8 \mathrm{ax}} \mathrm{H}\right), 1.90-2.04(\mathrm{~m}$, $\left.2 \mathrm{H}, \mathrm{C}_{2} \mathrm{H}\right), 1.10-1.30\left(\mathrm{~m}, 6 \mathrm{H}, \mathrm{C}_{5} \mathrm{CH}_{3}, \mathrm{Nlac} \beta \mathrm{CH}_{3}\right) ;{ }^{13} \mathrm{C} \mathrm{NMR}$ (100 $\left.\mathrm{MHz}, \mathrm{CDCl}_{3}\right) \delta 213.87,186.91,186.54,174.58$, $160.83,156.15,155.51,135.57,135.28,133.81,133.56$, $120.69,119.58,118.30,111.27,111.08,100.74,76.26$, $69.34,68.25,67.08,65.28,56.49,44.73,35.60,33.57$, $29.44,29.29,20.40,16.82 ; \mathrm{UV}\left(\mathrm{CH}_{3} \mathrm{OH}\right): \lambda_{\max }(\log \varepsilon)=234$ $(0.39), 253(0.24), 485(0.11)$; Mass $\left(\mathrm{FAB}^{+}, \mathrm{Na}\right) \mathrm{m} / \mathrm{z} 639$ (M $+\mathrm{Na})^{+}$.

Doxorubicin-14, 3'- $\mathrm{N}$-dilactate (5). The mixture of Llactic acid $(0.08 \mathrm{~mL}, 1.14 \mathrm{mmol})$ and EDCI $(0.22 \mathrm{~g}, 1.14$ mmol) in dry DMF $(50 \mathrm{~mL})$ was stirred on an ice bath for 30 $\mathrm{min}$ and allowed to warm to room temperature. To the stirred solution was added doxorubicin hydrochloride $(1 \mathrm{~b}, 0.30 \mathrm{~g}$, $0.46 \mathrm{mmol}$ ) and catalytic amounts of 4-pyrrolidinopyridine, and the mixture was stirred for $13 \mathrm{hr}$. The resulting mixture was diluted with $\mathrm{CH}_{2} \mathrm{Cl}_{2}(300 \mathrm{~mL})$, washed with water $(2 \times$ $200 \mathrm{~mL})$ and brine $(2 \times 200 \mathrm{~mL})$, dried over $\mathrm{MgSO}_{4}$, and the solvent was removed under reduced pressure. The residue was purified by column chromatography on silica gel $\left(\mathrm{CH}_{2} \mathrm{Cl}_{2} / \mathrm{Hexane} / \mathrm{CH}_{3} \mathrm{OH}=8: 2: 1\right)$ to give $5(0.28 \mathrm{~g}, 88 \%)$ as a red powder: $\mathrm{mp} 103-105^{\circ} \mathrm{C} ;[\alpha]_{\mathrm{D}}^{20}+145.29^{\circ}$ (c 0.1 , 
$\left.\mathrm{CH}_{3} \mathrm{OH}\right)$; IR (KBr) 3425, 2940, 1720, 1630, 1577, 1530, $1418,1280,1215,1124,985,782,705 \mathrm{~cm}^{-1}$; ${ }^{1} \mathrm{H}$ NMR $(400$ $\left.\mathrm{MHz}, \mathrm{CDCl}_{3}\right) \delta 13.98(\mathrm{~s}, 1 \mathrm{H}, \mathrm{PhOH}), 13.28(\mathrm{~s}, 1 \mathrm{H}, \mathrm{PhOH})$, $8.30(\mathrm{~d}, 1 \mathrm{H}, J=7.8 \mathrm{~Hz}, \mathrm{ArH}), 7.80$ (dd, $1 \mathrm{H}, J=7.8,8.3 \mathrm{~Hz}$, $\operatorname{ArH}), 7.41(\mathrm{~d}, 1 \mathrm{H}, J=8.3 \mathrm{~Hz}, \mathrm{ArH}), 7.00(\mathrm{~d}, 1 \mathrm{H}, J=8.3 \mathrm{~Hz}$, sugarNH), $5.52\left(\mathrm{~d}, 1 \mathrm{H}, J=5.8 \mathrm{~Hz}, \mathrm{C}_{4} \mathrm{H}\right), 5.38(\mathrm{~d}, 1 \mathrm{H}, J=$ $\left.18.1 \mathrm{~Hz}, \mathrm{C}_{14} \mathrm{H}\right), 5.30\left(\mathrm{~d}, 1 \mathrm{H}, J=18.1 \mathrm{~Hz}, \mathrm{C}_{14} \mathrm{H}\right), 5.21(\mathrm{~s}, 1 \mathrm{H}$, $\left.\mathrm{C}_{9} \mathrm{OH}\right), 4.89\left(\mathrm{~d}, 1 \mathrm{H}, J=4.1 \mathrm{~Hz}, \mathrm{C}_{7 \mathrm{eq}} \mathrm{H}\right), 4.76\left(\mathrm{~m}, 1 \mathrm{H}, \mathrm{C}_{1} \mathrm{H}\right)$, $4.30\left(\mathrm{~m}, 1 \mathrm{H}, \mathrm{C}_{1} \mathrm{H}\right), 4.20\left(\mathrm{q}, 1 \mathrm{H}, J=6.3 \mathrm{~Hz}, \mathrm{C}_{5} \mathrm{H}\right), 4.18(\mathrm{~m}$, $1 \mathrm{H}, \mathrm{Nlac} \alpha \mathrm{H}), 4.08\left(\mathrm{~s}, 3 \mathrm{H}, \mathrm{C}_{4} \mathrm{OCH}_{3}\right), 3.67\left(\mathrm{~m}, 1 \mathrm{H}, \mathrm{C}_{4} \mathrm{OH}\right)$, $3.45\left(\mathrm{~m}, 1 \mathrm{H}, \mathrm{C}_{3} \mathrm{H}\right), 3.27\left(\mathrm{~d}, 1 \mathrm{H}, J=18.5 \mathrm{~Hz}, \mathrm{C}_{10 \mathrm{eq}} \mathrm{H}\right), 2.99$ (d, $\left.1 \mathrm{H}, J=18.5 \mathrm{~Hz}, \mathrm{C}_{102 \mathrm{ax}} \mathrm{H}\right), 2.17$ (d, $1 \mathrm{H}, J=14.1 \mathrm{~Hz}$, $\left.\mathrm{C}_{8 \mathrm{eq}} \mathrm{H}\right), 2.13\left(\mathrm{dd}, 1 \mathrm{H}, J=4.1,14.1 \mathrm{~Hz}, \mathrm{C}_{8 \mathrm{ax}} \mathrm{H}\right), 1.75(\mathrm{~m}, 2 \mathrm{H}$, $\left.\mathrm{C}_{2} \mathrm{H}\right), 1.25\left(\mathrm{~m}, 9 \mathrm{H}, \mathrm{C}_{5} \cdot \mathrm{CH}_{3}, \mathrm{C}_{18} \mathrm{CH}_{3}\right.$, Nlac $\left.\mathrm{CH}_{3}\right) ;{ }^{13} \mathrm{C} \mathrm{NMR}$ $\left(100 \mathrm{MHz}, \mathrm{CDCl}_{3}\right) \delta 212.56,186.88,186.45,173.87$, $171.59,161.38,156.14,155.59,135.49,134.78,133.86$, $133.57,120.70,119.68,119.23,118.32,111.25,111.01$, $100.56,76.23,69.45,68.21,68.08,66.05,56.50,44.82$, $35.58,33.59,33.11,29.42,29.25,20.42,17.29$; UV $\left(\mathrm{CH}_{2} \mathrm{Cl}_{2}\right): \lambda_{\max }(\log \varepsilon)=252(0.24), 265(0.22), 485(0.11) ;$ Mass $\left(\mathrm{FAB}^{+}, \mathrm{Na}\right) \mathrm{m} / \mathrm{z} 711(\mathrm{M}+\mathrm{Na})^{+}$.

Daunomycin-14-stearate hydrochloride (6). 14-Bromodaunomycin hydrochloride (1c, $0.40 \mathrm{~g}, 0.62 \mathrm{mmol}$ ) and stearic acid $(0.35 \mathrm{~g}, 1.24 \mathrm{mmol})$ were dissolved in acetone $(300 \mathrm{~mL})$. To the mixture was added triethyl amine $(0.17$ $\mathrm{mL}, 1.24 \mathrm{mmol}$ ), and the mixture was then stirred at room temperature for $5 \mathrm{hr}$. After removing the solvent by a rotary evaporator, an etheral $\mathrm{HCl}$ in dry THF $(200 \mathrm{~mL})$ was added to the reaction mixture. The resulting mixture was stirred at $-20^{\circ} \mathrm{C}$ for $2 \mathrm{hr}$ and further stirred at room temperature for 3 $\mathrm{hr}$, and then the solvent was removed under reduced pressure. Purification of the residue by column chromatography $\left(\mathrm{CH}_{2} \mathrm{Cl}_{2} / \mathrm{CH}_{3} \mathrm{OH} / \mathrm{HCO}_{2} \mathrm{H} / \mathrm{H}_{2} \mathrm{O}=100: 15: 2: 1\right)$ gave daunomycin-14-stearate hydrochloride $(6,0.51 \mathrm{~g}, 97 \%)$ as a red powder: $\mathrm{mp} 100-102{ }^{\circ} \mathrm{C} ;[\alpha]_{\mathrm{D}}^{20}+112.04^{\circ}$ (c 0.1 , $\mathrm{CH}_{3} \mathrm{OH}$ ); IR (KBr) $3442,2927,2853,1743,1621,1582$, $1414,1286,1205,1118,984,815 \mathrm{~cm}^{-1} ;{ }^{1} \mathrm{H} N \mathrm{NR}(400 \mathrm{MHz}$, DMSO- $\left.d_{6}\right) \delta 13.86(\mathrm{~s}, 1 \mathrm{H}, \mathrm{PhOH}), 13.10(\mathrm{~s}, 1 \mathrm{H}, \mathrm{PhOH})$, $8.33\left(\mathrm{~s}, 2 \mathrm{H}, \mathrm{NH}_{2}\right), 7.87(\mathrm{~m}, 2 \mathrm{H}, \mathrm{ArH}), 7.67(\mathrm{~m}, 1 \mathrm{H}, \mathrm{ArH})$, $5.63\left(\mathrm{~d}, 1 \mathrm{H}, J=6.3 \mathrm{~Hz}, \mathrm{C}_{4} \mathrm{H}\right), 5.54\left(\mathrm{~s}, 1 \mathrm{H}, \mathrm{C}_{9} \mathrm{OH}\right), 5.32(\mathrm{~d}$, $\left.1 \mathrm{H}, J=18.5 \mathrm{~Hz}, \mathrm{C}_{1}+\mathrm{H}\right), 5.16\left(\mathrm{~d}, 1 \mathrm{H}, J=3.9 \mathrm{~Hz}, \mathrm{C}_{7 \mathrm{eq}} \mathrm{H}\right), 5.09$ $\left(\mathrm{d}, 1 \mathrm{H}, J=18.5 \mathrm{~Hz}, \mathrm{C}_{14} \mathrm{H}\right), 4.91\left(\mathrm{~m}, 1 \mathrm{H}, \mathrm{C}_{1} \cdot \mathrm{H}\right), 4.20(\mathrm{q}, 1 \mathrm{H}$, $\left.J=6.3 \mathrm{~Hz}, \mathrm{C}_{5} \mathrm{H}\right), 3.97\left(\mathrm{~s}, 3 \mathrm{H}, \mathrm{C}_{4} \mathrm{OCH}_{3}\right), 3.58(\mathrm{~m}, 1 \mathrm{H}$, $\left.\mathrm{C}_{4} \cdot \mathrm{OH}\right), 3.34\left(\mathrm{~m}, 1 \mathrm{H}, \mathrm{C}_{3} \mathrm{H}\right), 3.01(\mathrm{~d}, 1 \mathrm{H}, J=18.0 \mathrm{~Hz}$, $\left.\mathrm{C}_{10 \mathrm{eg}} \mathrm{H}\right), 2.86\left(\mathrm{~d}, 1 \mathrm{H}, J=18.0 \mathrm{~Hz}, \mathrm{C}_{102 \mathrm{x}} \mathrm{H}\right), 2.36(\mathrm{~m}, 2 \mathrm{H}$, $\left.\mathrm{C}_{16} \mathrm{H}\right), 2.26\left(\mathrm{~m}, 2 \mathrm{H}, \mathrm{C}_{8} \mathrm{H}\right), 1.52-1.92\left(\mathrm{~m}, 4 \mathrm{H}, \mathrm{C}_{2} \mathrm{H}, \mathrm{C}_{17} \mathrm{H}\right)$, $1.19\left(\mathrm{~m}, 31 \mathrm{H}, \mathrm{C}_{18-31} \mathrm{CH}_{2}, \mathrm{C}_{5} \mathrm{CH}_{3}\right), 0.82\left(\mathrm{~m}, 3 \mathrm{H}, \mathrm{C}_{32} \mathrm{CH}_{3}\right)$; ${ }^{13} \mathrm{C}$ NMR $\left(100 \mathrm{MHz}, \mathrm{DMSO}-d_{6}\right) \delta 208.01,186.30,185.48$, $173.42,174.41,165.34,160.79,135.67,135.09,134.20$, $133.84,121.34,119.94,118.99,114.10,110.60,99.42$, $75.13,69.56,66.24,65.45,56.59,46.50,36.11,35.24$, $34.43,33.43,31.38,31.30,30.99,29.69,29.67,29.63$, $29.51,29.47,29.37,29.14,29.05,28.72,28.32,24.44$, $22.55,22.10,16.68,13.94 ; \mathrm{UV}\left(\mathrm{CH}_{3} \mathrm{OH}\right): \lambda_{\max }(\log \varepsilon)=233$ $(0.81), 250(0.51), 482(0.19)$; Mass $\left(\mathrm{FAB}^{+}, \mathrm{Na}\right) \mathrm{m} / \mathrm{z} 833$ $(\mathrm{M}-\mathrm{HCl}+\mathrm{Na})^{+}$.

Daunomycin-3'- $N$-stearicarboamide (7). The mixture of stearic acid $(0.36 \mathrm{~g}, 1.27 \mathrm{mmol})$ and EDCI $(0.24 \mathrm{~g}, 1.27$ mmol) in dry DMF $(100 \mathrm{~mL})$ was stirred on an ice bath for $30 \mathrm{~min}$ and allowed to reach to room temperature. To the stirred solution was added daunomycin hydrochloride (1a, $0.60 \mathrm{~g}, 1.06 \mathrm{mmol}$ ) and catalytic amounts of 4-pyrrolidinopyridine, and the mixture was then stirred for $3 \mathrm{hr}$. The resulting mixture was diluted with $\mathrm{CH}_{2} \mathrm{Cl}_{2}(200 \mathrm{~mL})$, washed with water $(2 \times 200 \mathrm{~mL})$ and brine $(2 \times 200 \mathrm{~mL})$, dried over $\mathrm{MgSO}_{4}$, and the solvent was removed under reduced pressure. The residue was purified by column chromatography $\left(\mathrm{CH}_{2} \mathrm{Cl}_{2} / \mathrm{Hexane} / \mathrm{CH}_{3} \mathrm{OH}=12: 4: 1\right)$ to give daunomycin-3'- $N$-steariccarboamide $(7,0.88 \mathrm{~g}, 98 \%)$ as a red powder: $\mathrm{mp} 89-90^{\circ} \mathrm{C} ;[\alpha]_{\mathrm{D}}^{20}+142.02^{\circ}$ (c 0.1 , $\mathrm{CH}_{2} \mathrm{Cl}_{2}$ ); $\mathrm{IR}(\mathrm{KBr}) 3469,2939,2853,1738,1658,1621$, $1584,1547,1443,1412,1289,1209,1129,1129,1030,990$, $815 \mathrm{~cm}^{-1} ;{ }^{1} \mathrm{H}$ NMR $\left(400 \mathrm{MHz}, \mathrm{CDCl}_{3}\right) \delta 13.91(\mathrm{~s}, 1 \mathrm{H}$, $\mathrm{PhOH}$ ), 13.61 (s, 1H, PhOH), 7.95 (d, $1 \mathrm{H}, J=7.8 \mathrm{~Hz}, \mathrm{ArH}$ ), 7.72 (dd, $1 \mathrm{H}, J=7.8,8.3 \mathrm{~Hz}, \mathrm{ArH}), 7.33(\mathrm{~d}, 1 \mathrm{H}, J=8.3 \mathrm{~Hz}$, $\mathrm{ArH}), 5.51$ (d, $\left.1 \mathrm{H}, J=6.8 \mathrm{~Hz}, \mathrm{C}_{4} \mathrm{H}\right), 5.29(\mathrm{~s}, 1 \mathrm{H}, \mathrm{C} 9 \mathrm{OH}$ ), $5.18\left(\mathrm{~d}, 1 \mathrm{H}, J=4.4 \mathrm{~Hz}, \mathrm{C}_{7 \mathrm{eq}} \mathrm{H}\right), 5.14\left(\mathrm{~m}, 1 \mathrm{H}, \mathrm{C}_{1} \mathrm{H}\right), 4.26(\mathrm{~m}$, $\left.1 \mathrm{H}, \mathrm{C}_{3} \mathrm{H}\right), 4.09\left(\mathrm{q}, 1 \mathrm{H}, J=6.8 \mathrm{~Hz}, \mathrm{C}_{\mathrm{r}} \mathrm{H}\right), 4.02(\mathrm{~s}, 3 \mathrm{H}$, $\left.\mathrm{C}_{4} \mathrm{OCH}_{3}\right), 3.61\left(\mathrm{~m}, 1 \mathrm{H}, \mathrm{C}_{4} \mathrm{OH}\right), 3.33\left(\mathrm{~m}, 1 \mathrm{H}, \mathrm{C}_{3} \mathrm{H}\right), 3.13(\mathrm{~d}$, $\left.1 \mathrm{H}, J=18.5 \mathrm{~Hz}, \mathrm{C}_{10 \mathrm{eg}} \mathrm{H}\right), 2.80\left(\mathrm{~d}, 1 \mathrm{H}, J=18.5 \mathrm{~Hz}, \mathrm{C}_{10 \mathrm{ax}} \mathrm{H}\right)$, $2.38\left(\mathrm{~s}, 3 \mathrm{H}, \mathrm{C}_{14} \mathrm{CH}_{3}\right), 2.24\left(\mathrm{~d}, 1 \mathrm{H}, J=14.6 \mathrm{~Hz}, \mathrm{C}_{8 \mathrm{eq}} \mathrm{H}\right), 2.08$ (dd, $1 \mathrm{H}, J=4.4,14.6 \mathrm{~Hz}, \mathrm{C}_{8 \mathrm{ax}} \mathrm{H}$ ), $2.01\left(\mathrm{~m}, 2 \mathrm{H}, \mathrm{NstC}_{2} \mathrm{CH}_{2}\right.$ ), $1.80\left(\mathrm{~m}, 2 \mathrm{H}, \mathrm{NstC}_{3} \mathrm{CH}_{2}\right), 1.39-1.64\left(\mathrm{~m}, 2 \mathrm{H}, \mathrm{C}_{2} \mathrm{H}\right), 1.20(\mathrm{~m}$, $\left.28 \mathrm{H}, \mathrm{NstC}_{4-1} 7 \mathrm{CH}_{2}\right), 1.12\left(\mathrm{~d}, 3 \mathrm{H}, J=6.3 \mathrm{~Hz}, \mathrm{C}_{5} \mathrm{CH}_{3}\right), 0.84$ $\left(\mathrm{m}, 3 \mathrm{H}, \mathrm{NstC}_{18} \mathrm{CH}_{3}\right) ;{ }^{13} \mathrm{C} \mathrm{NMR}\left(100 \mathrm{MHz}, \mathrm{CDCl}_{3}\right) \delta 212.27$, $186.92,186.46,173.39,172.29,160.89,156.38,155.77$, $135.63,135.42,134.50,133.94,120.74,119.75,118.28$, $111.30,111.12,100.67,76.53,70.49,69.99,66.29,56.58$, $43.67,36.62,35.13,34.22,33.32,31.88,31.54,30.46$, $29.66,29.62,29.50,29.44,29.33,29.27,29.18,25.53$, $25.20,24.90,22.65,22.61,16.94,14.08 ; \mathrm{UV}\left(\mathrm{CH}_{2} \mathrm{Cl}_{2}\right): \lambda_{\max }$ $(\log \varepsilon)=235(0.33), 252(0.21), 485(0.10)$; Mass $\left(\mathrm{FAB}^{+}\right.$, Na) $\mathrm{m} / \mathrm{z} 863(\mathrm{M}+\mathrm{Na})^{+}$.

Doxorubicin-3' $\mathrm{N}$-steariccarboamide (8). The mixture of stearic acid $(0.41 \mathrm{~g}, 1.45 \mathrm{mmol})$ and EDCI $(0.28 \mathrm{~g}, 1.45$ mmol) in dry DMF $(100 \mathrm{~mL})$ was stirred on an ice bath for $30 \mathrm{~min}$ and allowed to reach to room temperature. To the stirred solution was added doxorubicin hydrochloride (1), $0.70 \mathrm{~g}, 1.21 \mathrm{mmol}$ ) and catalytic amounts of 4-pyrrolidinopyridine, and the mixture was then stirred for $3 \mathrm{hr}$. The resulting mixture was diluted with $\mathrm{CH}_{2} \mathrm{Cl}_{2}(300 \mathrm{~mL})$, washed with water $(2 \times 300 \mathrm{~mL})$ and brine $(2 \times 300 \mathrm{~mL})$, dried over $\mathrm{MgSO}_{4}$, and the solvent was removed under reduced pressure. The residue was purified by column chromatography $\left(\mathrm{CH}_{2} \mathrm{Cl}_{2} / \mathrm{Hexane} / \mathrm{CH}_{3} \mathrm{OH}=12: 4: 1\right)$ to give doxorubicin- $3^{\prime}-\mathrm{N}$-steariccarboamide $(\mathbf{8}, 0.96 \mathrm{~g}, 98 \%)$ as a pale red powder: $\mathrm{mp} 97-99^{\circ} \mathrm{C}$; $[\alpha]_{\mathrm{D}}^{20}+97.01^{\circ}$ (c 0.1 , $\mathrm{CH}_{2} \mathrm{Cl}_{2}$ ); IR ( $\left.\mathrm{KBr}\right) 3482,2927,2853,2738,1627,1584$, $1449,1412,1289,1209,1123,1018,987,815,790 \mathrm{~cm}^{-1} ;{ }^{1} \mathrm{H}$ NMR (400 MHz, $\left.\mathrm{CDCl}_{3}\right) \delta 13.80(\mathrm{~s}, 1 \mathrm{H}, \mathrm{PhOH}), 13.00(\mathrm{~s}$, $1 \mathrm{H}, \mathrm{PhOH}), 7.87$ (d, $1 \mathrm{H}, J=7.8 \mathrm{~Hz}, \mathrm{ArH}), 7.65$ (dd, $1 \mathrm{H}, J=$ $7.8,8.3 \mathrm{~Hz}, \mathrm{ArH}), 7.25(\mathrm{~d}, 1 \mathrm{H}, J=8.3 \mathrm{~Hz}, \mathrm{ArH}), 5.86(\mathrm{~d}$, $\left.1 \mathrm{H}, J=6.8 \mathrm{~Hz}, \mathrm{C}_{4} \mathrm{H}\right), 5.37\left(\mathrm{~s}, 1 \mathrm{H}, \mathrm{C}_{9} \mathrm{OH}\right), 5.07(\mathrm{~d}, 1 \mathrm{H}, J=$ $\left.4.4 \mathrm{~Hz}, \mathrm{C}_{7 \mathrm{eg}} \mathrm{H}\right), 4.98\left(\mathrm{~m}, 1 \mathrm{H}, \mathrm{C}_{1} \cdot \mathrm{H}\right), 4.58\left(\mathrm{~s}, 2 \mathrm{H}, \mathrm{C}_{1+} \mathrm{H}\right), 4.01-$ 
$4.12(\mathfrak{q}, 1 \mathrm{H}, J=6.8 \mathrm{~Hz}, \mathrm{C}: \mathrm{H}), 3.92\left(\mathrm{~s}, 3 \mathrm{H}, \mathrm{C}_{4} \mathrm{OCH}_{3}\right), 3.53$ $\left(\mathrm{m}, 1 \mathrm{H}, \mathrm{C}_{4} \mathrm{OH}\right), 3.23\left(\mathrm{~m}, 1 \mathrm{H}, \mathrm{C}_{3} \mathrm{H}\right), 3.09(\mathrm{~d}, 1 \mathrm{H}, J=18.5$ $\left.\mathrm{Hz}, \mathrm{C}_{10 \mathrm{eq}} \mathrm{H}\right), 2.75\left(\mathrm{~d}, 1 \mathrm{H}, J=18.5 \mathrm{~Hz}, \mathrm{C}_{100 \mathrm{x}} \mathrm{H}\right), 2.36(\mathrm{~d}, 1 \mathrm{H}, J$ $\left.=14.6 \mathrm{~Hz}, \mathrm{C}_{8 \mathrm{eq}} \mathrm{H}\right), 2.00\left(\mathrm{dd}, 1 \mathrm{H}, J=4.4,14.6 \mathrm{~Hz}, \mathrm{C}_{82 \mathrm{x}} \mathrm{H}\right)$, $1.95\left(\mathrm{~m}, 2 \mathrm{H}, \mathrm{NstC}_{2} \mathrm{CH}_{2}\right), 1.72\left(\mathrm{~m}, 2 \mathrm{H}, \mathrm{C}_{2} \mathrm{H}\right), 1.60(\mathrm{~m}, 2 \mathrm{H}$, $\left.\mathrm{NstC}_{3} \mathrm{CH}_{2}\right), 1.11-1.23\left(\mathrm{~m}, 31 \mathrm{H}, \mathrm{C}_{5} \mathrm{CH}_{3}, \mathrm{NstC}_{41} \mathrm{CH}_{2}\right), 0.78$ $\left(\mathrm{m}, 3 \mathrm{H}, \mathrm{NstC}_{18} \mathrm{CH}_{3}\right) ;{ }^{13} \mathrm{C} \mathrm{NMR}\left(100 \mathrm{MHz}, \mathrm{CDCl}_{3}\right) \delta 206.74$, $186.74,186.27,173.18,172.54,160.83,156.18,155.56$, $135.63,135.28,133.88,133.64,120.57,119.71,118.32$, $111.29,111.11,100.63,77.06,69.73,69.61,67.25,65.84$, $56.48,45.07,36.71,33.87,33.43,31.87,31.53,29.86$, $29.65,29.64,29.60,29.45,29.31,29.26,29.22,29.08$, $25.66,24.90,22.63,22.60,16.66,14.07$; UV $\left(\mathrm{CH}_{2} \mathrm{Cl}_{2}\right): \lambda_{\max }$ $(\log \varepsilon)=235(0.37), 252(0.22), 482(0.10)$; Mass $\left(\mathrm{FAB}^{+}\right.$, $\mathrm{Na}) \mathrm{m} / \mathrm{z} 833(\mathrm{M}+\mathrm{Na})^{+}$.

Doxorubicin-14, 3'-disteariccarboamide (9). The mixture of stearic acid $(0.75 \mathrm{~g}, 2.65 \mathrm{mmol})$ and EDCI $(0.58 \mathrm{~g}$, $3.02 \mathrm{mmol}$ ) in dry DMF $(100 \mathrm{~mL})$ was stirred in an ice bath for $30 \mathrm{~min}$ and allowed to reach to room temperature. To the stirred solution was added doxorubicin hydrochloride $(\mathbf{1 b}$, $0.70 \mathrm{~g}, 1.21 \mathrm{mmol}$ ) and catalytic amounts of 4-pyrrolidinopyridine, and the mixture was stirred for $8 \mathrm{hr}$. The resulting mixture was extracted with $\mathrm{CH}_{2} \mathrm{Cl}_{2}(300 \mathrm{~mL})$, washed with water $(2 \times 300 \mathrm{~mL})$ and brine $(2 \times 300 \mathrm{~mL})$, dried over $\mathrm{MgSO}_{4}$, and the solvent was removed under reduced pressure. The residue was purified by column chromatography on silica gel $\left(\mathrm{CH}_{2} \mathrm{Cl}_{2} / \mathrm{Hexane} / \mathrm{CH}_{3} \mathrm{OH}=12: 6: 1\right)$ to give compound $9(1.12 \mathrm{~g}, 86 \%)$ as a pale red solid: $\mathrm{mp} 82-84^{\circ} \mathrm{C}$; $[\alpha]_{\mathrm{D}}^{20}+187.05^{\circ}\left(\mathrm{c} 0.1, \mathrm{CH}_{2} \mathrm{Cl}_{2}\right) ; \mathrm{IR}(\mathrm{KBr}) 3494,2927,2853$, $1738,1640,1584,1541,1467,1418,1289,1215,1123$, $1018,987 \mathrm{~cm}^{-1} ;{ }^{1} \mathrm{H} \mathrm{NMR}\left(400 \mathrm{MHz}, \mathrm{CDCl}_{3}\right) \delta 13.93(\mathrm{~s}, 1 \mathrm{H}$, $\mathrm{PhOH}$ ), 13.16 (s, 1H, $\mathrm{PhOH}$ ), 7.99 (d, $1 \mathrm{H}, J=7.8 \mathrm{~Hz}, \mathrm{ArH}$ ), 7.74 (dd, $1 \mathrm{H}, J=7.8,8.3 \mathrm{~Hz}, \mathrm{ArH}), 7.35(\mathrm{~d}, 1 \mathrm{H}, J=8.3 \mathrm{~Hz}$, $\mathrm{ArH}), 5.53\left(\mathrm{~d}, 1 \mathrm{H}, J=6.8 \mathrm{~Hz}, \mathrm{C}_{4} \mathrm{H}\right), 5.29(\mathrm{~s}, 1 \mathrm{H}, \mathrm{C} \mathrm{OH})$, $5.24\left(\mathrm{~d}, 1 \mathrm{H}, J=18.0 \mathrm{~Hz}, \mathrm{C}_{14} \mathrm{H}\right), 5.18(\mathrm{~d}, 1 \mathrm{H}, J=18.0 \mathrm{~Hz}$, $\left.\mathrm{C}_{14} \mathrm{H}\right), 5.07\left(\mathrm{~d}, 1 \mathrm{H}, J=4.4 \mathrm{~Hz}, \mathrm{C}_{7 \mathrm{eq}} \mathrm{H}\right), 4.51\left(\mathrm{~m}, 1 \mathrm{H}, \mathrm{C}_{1} \mathrm{H}\right)$, $4.12(\mathrm{q}, 1 \mathrm{H}, J=6.8 \mathrm{~Hz}, \mathrm{C}: \mathrm{H}), 4.09\left(\mathrm{~s}, 3 \mathrm{H}, \mathrm{C}_{4} \mathrm{OCH}_{3}\right), 3.62$ $\left(\mathrm{m}, 1 \mathrm{H}, \mathrm{C}_{+} \mathrm{OH}\right), 3.24\left(\mathrm{~m}, 1 \mathrm{H}, \mathrm{C}_{3} \mathrm{H}\right), 3.23(\mathrm{~d}, 1 \mathrm{H}, J=19.0$ $\left.\mathrm{Hz}, \mathrm{C}_{10 \mathrm{eg}} \mathrm{H}\right), 2.94\left(\mathrm{~d}, 1 \mathrm{H}, J=19.0 \mathrm{~Hz}, \mathrm{C}_{100 \mathrm{x}} \mathrm{H}\right), 2.43(\mathrm{~d}, 1 \mathrm{H}, J$ $\left.=14.6 \mathrm{~Hz}, \mathrm{C}_{8 \mathrm{eq}} \mathrm{H}\right), 2.14\left(\mathrm{dd}, 1 \mathrm{H}, J=4.4,14.6 \mathrm{~Hz}, \mathrm{C}_{8 \mathrm{\alpha x}} \mathrm{H}\right)$, $2.02\left(\mathrm{~m}, 4 \mathrm{H}, \mathrm{C}_{16} \mathrm{CH}_{2}, \mathrm{NstC}_{2} \mathrm{CH}_{2}\right), 1.82\left(\mathrm{~m}, 2 \mathrm{H}, \mathrm{C}_{2} \mathrm{H}\right), 1.66$ $\left(\mathrm{m}, 4 \mathrm{H}, \mathrm{C}_{17} \mathrm{CH}_{2}, \mathrm{NstC}_{3} \mathrm{CH}_{2}\right), 1.05-1.40\left(\mathrm{~m}, 59 \mathrm{H}, \mathrm{C}_{5} \mathrm{CH}_{3}\right.$. $\left.\mathrm{C}_{18-31} \mathrm{CH}_{2}, \mathrm{NstC}_{4-17} \mathrm{CH}_{2}\right), 0.84\left(\mathrm{~m}, 6 \mathrm{H}, \mathrm{C}_{32} \mathrm{CH}_{3}, \mathrm{NstC}_{18} \mathrm{CH}_{3}\right.$ ); ${ }^{13} \mathrm{C}$ NMR $\left(100 \mathrm{MHz}, \mathrm{CDCl}_{3}\right) \delta 206.79,18.05,186.52$, $182.99,174.02,173.44,173.20,172.34,160.98,156.24$, $155.78,149.64,135.71,135.47,135.07,134.07,133.62$, $132.56,120.79,119.83,118.32,118.24,113.37,111.42$, $111.25,100.61,95.42,77.11,75.14,75.02,70.56,69.77$, $66.52,65.90,60.37,56.62,43.69,36.61,35.52,34.24$, $33.88,33.58,31.90,31.56,30.46,29.68,29.64,29.52$, $29.45,29.34,29.27,29.18,29.09,25.50,25.23,24.90$, $22.67,22.63,21.64,21.02,16.93,14.17,14.09$; UV
$\left(\mathrm{CH}_{2} \mathrm{Cl}_{2}\right): \lambda_{\max }(\log \varepsilon)=235(0.36), 252(0.22), 482(0.11)$ Mass $\left(\mathrm{FAB}^{+}, \mathrm{Na}\right) \mathrm{m} / \mathrm{z} 1099(\mathrm{M}+\mathrm{Na})^{+}$.

Acknowledgement. We gratefully acknowledged KBSI for the NMR and mass analyses.

\section{References}

1. Priebe, W, Anthracycline Antibiotics, New Analognes, Methods of Delivery; and Mechanisms of Action; American Chemical Society: Washington DC, 1995.

2. Mettler, F. P.; Young, D. M.; Ward, J. M. Cancer Res. 1977, 37 , 2705 .

3. Hortobagyi, G. N. Dritgs 1997, $54, \mathrm{I}$.

4. Levin, Y.; Sela, B.-A. US Patent 4256632, 1981

5. Lee, M. G; Yang J. Drugs of the Futume 1996, 21, 782.

6. Arcamone, F.; Franceschi, G.; Minghetti, A.; Penco, S.; Redaelli, S. J. Med. Chem. 1974, 17, 335.

7. Arcamone, F.; Franceschi, G.; Penco, S. US Patent $3803 / 24$. 1974.

8. Rho, Y. S.; Kim, W. -J.; Park, \$. H.; Yoo, D. J.; Kang, H. S.; Chung, S. R. Bull. Korean Chem. Soc. 2001, 22, 581.

9. Rho, Y. S.; Kim, G. I.; Kim, W.-J.; Park, S. H.; Yoo, D. J.; Kang, H. S.; Chung, S. R. Bull. Korean Chem. Soc. 2001, 22, 587.

10. Rho, Y. S.; Kim, W.J.; Park, S. H.; Yoo, D. J.; Kang, H. S.; Chung, S. R. Bull. Korean Chem. Sac. 2001, 22, 963.

I1. Rho, Y. S.; Park, S. H.; Kim, W.-J.; Kim, G. I.; Yoo, D. J.; Kang, H. S.; Chung, S. R. Synth. Commun. 2002, 32(13), 1961.

12. Rho, Y. S.; Park, J.; Kim, G.; Kim, H.; Sin, H.; Suh, P. W.; Yoo, D. J. Synth. Commin. 2004, 34(9), 1703.

13. Rho, Y. S.; Kim, S. Y.; Kim, W.-J.; Yun, Y. K.; Sin, H. S.; Yoo, D. J. Syth. Commun. 2004, 34(19), 3497.

14. Lavie, Y.; Cao, H.-T.; Bursten, S. L.; Giuliano, A. E.; Cabot, M. C. J. Biological Chem, 1996, 27/(32), 19530.

15. Hauser, F. M.; Hewawasam, P; Rho, Y. S. J. Org. Chem. 1989, 54,5110 .

16. Rho, Y. S.; Park, S.; Kim, S. Y.; Cho, I.; Lee, C.; Kang, H. S.; Cheong, C. Bull. Korean Chem. Soc. 1998, $19,74$.

17. Rho, Y. S.; Kim, S. Y.; Cho, I.; Kang, H. S.; Yoo, D. J.; Cheong, C. Bthl. Korean Chem. Soc, 1998, 19, 1059.

18. Rho, Y. S.; Choi, Y.; Kim, G.; Sin, H.; Yoo, D. J.; Kim, S. H.; Cheong, C. Bull. Korean Chem. Soc. 1999, 20,55I.

19. Rho, Y. S.; Ko, H. K.; Kim, W.-J.; Yoo, D. J.; Kang, H. S. Bull. Korean Chem. Soc. 2000, 21, 774.

20. Rho, Y. S.; Ko, H. K.; Sin, H.; Yoo, D. J. Bull. Korean Chem. Soc. 1999, 20, 1517.

2I. Rho, Y. S.; Park, S. C.; Kang, H. S. Novel Anthracycline Derivatives and Their Preparation. US Patent $(09 / 674,673)$, EU Patent (99 919679.3), JPN Patent (2000-547095), KOR Patent (2000-7012427).

22. Umezawa, H.; Takeuchi, T.; Naganawa, H.; Tatsuta, K. US Patent 4360664,1982 .

23. Ducep, J.-B.; Farge, D.; Ponsinet, G.; Reisdorf, D. US Patent 4225589,1980 .

24. (a) Dhaon, M. K.; Olsen, R. K.; Ramasamy, K. J. Org. Chen. 1982, 47, 1962. (b) Hudson, D. J. Org. Chem. 1988, 53,617.

25. Desai, M. C.; Stephens Stramiello, L. M. Tetrahedron Lett. 1993, 34,7685 .

26. Akaji, K.; Kuriyama, N.; Kiso, Y. Tetrahedron Lett, 1994, 35, 3315.

27. Armerigo, W. L. F.; Perrin, D. D. Purification of Laboratory: Chenicals, 4th Ed.; Oxford: Butterworth-Heinemann, 1996. 